\title{
Bioengineered Periosteal Progenitor Cell Sheets to Enhance Tendon-Bone Healing in A Bone Tunnel
}

\author{
Chih-Hsiang Chang ${ }^{1}$, Chih-Hwa Chen ${ }^{1}$, Hsia-Wei Liu², Shu-Wen Whu ${ }^{1}$, Shih-Hui Chen", \\ Ching-Lin Tsai ${ }^{3}$, Ging-Ho Hsiue ${ }^{4}$
}

Background: Tendon-bone tunnel healing is crucial for long term success in anterior cruciate ligament (ACL) reconstruction. The periosteum contains osteochondral progenitor cells that can differentiate into osteoblasts and chondroblasts during tendon-bone healing. We developed a scaffold-free method using polymerized fibrin-coated dishes to make functional periosteal progenitor cell (PPC) sheets. Bioengineered PPC sheets for enhancing tendon-bone healing were evaluated in an extra-articular bone tunnel model in rabbit.

Methods: $\quad$ PPC derived from rabbit tibia periosteum, cultivated on polymerized fibrin-coated dishes and harvested as PPC sheet. A confocal microscopy assay was used to evaluate the morphology of PPC sheets. PPC sheets as a periosteum to wrap around hamstring tendon grafts were pulled into a 3-mm diameter bone tunnel of tibia, and compared with a tendon graft without PPC sheets treatment. Rabbits were sacrificed at 4 and 8 weeks postoperatively for biochemical assay and histological assay to demonstrate the enhancement of PPC sheets in tendon-bone healing.

Results: $\quad$ PPC spread deposit on fibrin on the dish surface with continuous monolayer PPC was observed. Histological staining revealed that PPC sheets enhance collagen and glycosaminoglycans deposition with fibrocartilage formation in

At a Glance Commentary

Scientific background of the subject

Tendon-bone tunnel healing is one of the key factors for successful anterior cruciate ligament reconstruction. For better and quicker tendon-bone healing, many materials have been used to augment or enhance the tendon-bone healing process. A bioengineered periosteal progenitor cell sheet without scaffolds was created to enhance tendon-bone tunnel healing.

\section{What this study adds to the field}

Periosteal progenitor cell monolayer maintains the differentiated and osteochondral potential to promote fibrocartilage and bone formation in tendon-bone junction. A periosteal progenitor cell sheet was developed to promote tendon-bone interface integration and healing. Bioengineered periosteal progenitor cell sheets provide a novel strategy to enhance tendon-bone healing in anterior cruciate ligament reconstruction. the tendon-bone junction at 4 weeks. Collagen fiber with fibrocartilage formation at tendon-bone junction was also found at 8 weeks. Matured fibrocartilage and dense collagen fiber were formed at the tendon-bone interface at 8 weeks by Masson trichrome and Safranin-O staining.

Conclusions: Periosteal progenitor cell monolayer maintains the differentiated capacity and osteochondral potential in order to promote fibrocartilage formation in tendon-bone junction. Bioengineered PPC sheets can offer a new feasible therapeutic strategy of a novel approach to enhance tendon-bone junction healing.

(Biomed J 2012;35:473-80)

Key words: cell sheet, periosteum, tendon-bone healing

From the ${ }^{1}$ Department of Orthopaedic Surgery, Chang Gung Memorial Hospital at Keelung, Chang Gung University College of Medicine, Taoyuan, Taiwan, ${ }^{2}$ Life Science, Fu Jen Catholic University, New Taipei City, Taiwan, ${ }^{3}$ Departments of Orthopaedic Surgery, National Taiwan University, Taipei, Taiwan, ${ }^{4}$ Departments of Chemical Engineering, Chung Yuan University, Taoyuan, Taiwan

Received: Aug. 31, 2011; Accepted: Apr. 9, 2012

Correspondence to: Dr. Chih-Hwa Chen, Department of Orthopaedic Surgery, Chang Gung Memorial Hospital at Keelung. No.222, Maijin Rd., Anle District, Keelung City 204, Taiwan (R.O.C.). Tel: 886-2-24313131 ext. 2613; Fax: 886-2-24332655; E-mail: afachen@ doctor.com

DOI: $10.4103 / 2319-4170.104412$ 
A nterior cruciate ligament (ACL) reconstruction using semitendinosus and gracilis tendon grafts is popular in orthopedic surgery. Successful ligament reconstruction necessitates effective osteointegration of tendon grafts. ${ }^{[1]} \mathrm{In}$ the early period after ACL reconstruction, the tendon-bone interface is weak and is formed by woven bone formation..$^{[2]}$ The interface between hamstring tendon graft and bone tunnel is composed of three distinct histological zones and resembles a fibrous tendon insertion, which means the graft and bone tunnel is a weak link, and may result in reconstruction failure. ${ }^{[3]}$ To protect and ensure tendon graft healing in the bone tunnel, rehabilitation, range of motion (ROM), and other specific activities are often delayed ${ }^{[4]}$ For better and quicker tendon-bone healing, many materials have been used to augment or enhance the tendon-bone healing process; such as bone morphogenetic protein-2 (BMP-2), transforming growth factor (TGF), fibroblast growth factor (FGF), bone marrow stromal cells (BMSCs), calcium phosphate, mesenchymal stem cells (MSCs), BMP-2 gene transfer, synovium-derived stem cell (SDSCs), and the periosteum. ${ }^{[5-15]}$

The periosteum is a bilayered tissue that posed between the overlying soft tissue and cortical bone. There is an outer fibrous layer and an inner cambium layer, and the fibrous layer contains fibroblasts while the cambium layer consists of chondroprogenitor and osteoprogenitor cells that have the ability to differentiate into cartilage and bone ${ }^{[11-15]}$ Success of tendon reconstruction is the bone ingrowth into the tendon. ${ }^{[1,11]}$ Previous studies revealed that periosteal tissue may enhance healing of the interface between the tendon and the bone by forming fibrocartilage and calcified fibrocartilage. ${ }^{[16,17]}$ In clinical study, periosteum could enhance tendon-bone healing in ACL reconstruction with satisfactory clinical outcome. ${ }^{[18]}$ The progenitor cells of periosteal cambial layer (PPC) may be used to enhance the healing process.

Biomimetic materials with scaffolds-free cell sheet had been developed by Okano et al., in 1993. ${ }^{[19]}$ This technique allows for the noninvasive harvest of cultured cells as an intact sheet with their formatted extracellular matrix. This simple method of cell sheet engineering may also be applied for various other cell types. ${ }^{[20,21]}$

We use the biodegradable polymerized fibrin-coated dishes to make functional PPC sheets as biological periosteal tissue in orthopedic regeneration. The purpose of this study is to determine the effect of bioengineered periosteal progenitor cell sheets on tendon-bone healing. We hypothesize that the PPC sheets could serve as effective materials like periosteum to promote fibrocartilage formation and enhance tendon-bone healing in a tibia bone tunnel. We use histological examine including Hematoxylin-eosin (H-E), Masson's trichrome (M-T), and Safranin-O staining to evaluate the interface between tendon graft and bone tunnel.

\section{METHODS}

\section{Isolation of PPC}

PPC were obtained by stripping the periosteum from the tibia of mature New Zealand white rabbits. The harvested periosteum was placed in a $0.25 \%$ trypsin solution and $0.1 \%$ ethylene-diamine-tetra-acetic acid (EDTA) for $30 \mathrm{~min}$ at $37 \mathrm{C}$ and shaken in $1 \mathrm{mg} / \mathrm{mL}$ of type I collagenase digestive solution for $90 \mathrm{~min}$ at $37 \mathrm{C}$. After washing and centrifugation, the pellets were resuspended in high-glucose Dulbecco's modified Eagle's medium (DMEM, Gibco BRL; Life Technologies, Gaithersburg, MD) supplemented with $10 \%$ fetal bovine serum (FBS, HyClone, South Logan, UT) and 1\% penicillin-streptomycin (Biological Industries, Kibbutz Beit Haemek, Israel). PPC were finely plated in a T25 culture flask (Corning Inc., Acton, MA, USA). Nonadherent cells were removed by changing the fresh medium after cultured for 5 days. The culture medium was replenished every 3 days.

\section{Remove fibroblast from culture medium}

We use partial trypsinization to remove fibroblast cells. This was usually performed during the cell passage. First, the media was vacuumed off. Then the cells were washed with PBS, the $0.05 \%$ trypsin solution was added, and the cells were incubated for 5 minutes at $37 \mathrm{C}, 5 \% \mathrm{CO}_{2}$. This caused the fibroblasts to change shape and lift off the plate. The trypsin and fibroblasts were vacuumed off and the cells were washed with PBS once more. Both procedures were done at least four times until the cells were devoid of fibroblasts, leaving only PPC in culture.

\section{Preparation of PPC sheet}

Thrombin (Sigma-Aldrich, Louis, USA) was added into $100 \mathrm{mM} \mathrm{CaCl}_{2}$ solution (Sigma-Aldrich) to obtain $100 \mathrm{U} / \mathrm{ml}$. Then solution was filtered through $0.22 \mu \mathrm{m}$ filter, and mixed with phosphate buffer solution (PBS, $\mathrm{pH}=7.4$ ) to form $50 \mathrm{U} / \mathrm{ml}$. Fibrinogen (Sigma- Aldrich) was added into PBS to obtain $12.5 \mathrm{mg} / \mathrm{ml}$. Thrombin solution was mixing with fibrinogen solution at equal volume. The mixture was replaced into a diameter $6-\mathrm{cm}$ culture dish containing a diameter $4.7-\mathrm{cm}$ silicon O-ring. The culture dish was kept at room temperature for 2 hours. $5 \times 10^{[5]} \mathrm{PPC}$ were seeded into a fibrin coating dish, and then incubated under the same conditions for primary cell cultures as previously mentioned. The culture medium contain aprotinin 350KIU/ $\mathrm{ml}$ (Protech Technology Enterprise Co. Ltd., Taiwan) was replaced every 3 days. The aprotinin was used to inhibit protease secreted by cells which will digest the fibrin. After culture for 10 days, the aprotinin was removed and the cell became to secrete protease. The silicon O-ring was then 
taken out and due to weakened fibrin, a cell sheet could be harvested by a scraper [Figure 1(A)]. Some fibrin was remained on cell sheet, but due to small amount and was rapid digested by cell in $1 \sim 2$ days in vivo, the fibrin has no structural function on cell sheet. A confocal microscopy assay and immunofluorescence staining were used to evaluate cell distribution and sheet continuity.

\section{Immunofluorescence staining}

We rinse the sections in PBS for 4 min, and then block the specimen in Blocking Buffer for 60 minutes. The specimen was incubated in Fibrin (monosan, MON 5019) for 1 hour at room temperature. Then the specimen was rinsed three times in PBS for 5 minutes each. The specimen was incubated in fluorochrome-conjugated secondary antibody for 1 hours at room temperature in dark. Then the specimen was rinsed three times in PBS for 5 minutes each. $\alpha$-SMA Cy3(sigma, C 6198) was used for cellular structure. The specimen was rinsed three times in PBS for 5 minutes each. Counterstain with DAPI for 20 minutes at room temperature was for cellular nucleus. The specimen was rinsed three times in PBS for 5 minutes each. Then the coverslip with anti-fade fluorescent mounting medium was sealed with nail polish. The slides were stored in dark at $4 \mathrm{C}$.

\section{Animal study design and surgery}

Forty skeletally mature New Zealand white rabbits (mean weight $3.5 \mathrm{~kg}$ ) were used in this study. Animals were randomly assigned for PPC and control group, with each group consisting of 20 rabbits. Animals in Each group were randomly assigned for 4 and 8 week group postoperation. Animal treatment conformed to the Guidelines for Care and Use of Laboratory Animals and was approved by the Committee of Experimental Animal Sciences.

Ketamine ( $40 \mathrm{mg} / \mathrm{kg}$ ) with xylazine $(5 \mathrm{mg} / \mathrm{kg}$ ) (Rompun; Bayer Healthcare, Leverkusen, Germany) was intramuscularly injected to induce general anesthesia. The rabbits underwent an operative procedure of the

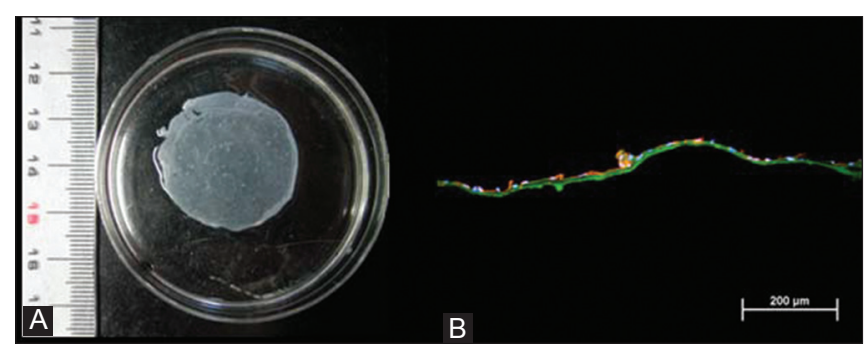

Figure 1: Periosteal progenitor cell sheet cultured for 10 days in vitro. (A) The appearance: a diameter $30-\mathrm{mm}$ circular cell sheet; (B) Confocal microscopy assay: periosteal progenitor cells continuously spread on fibrin and monolayer formation. The thickness of cell sheet was about $20 \mu \mathrm{m}$. Red: a-Actin; Blue: Nucleus; Green: Fibrin. extra-articular tendon-bone healing model in which no mechanical loading occurred at the rerouted long digital extensor tendon. Using an aseptic approach [Figure 2(A)], the knee joint was accessed via a lateral parapatellar incision. The long digital extensor tendon was identified and then detached from its insertion on the lateral femoral condyle by sharp dissection [Figures 2(B) and (C)]. The free tendon was sutured using 3 - 0 Vicryl suture (Ethicon, Somerville, NJ) [Figure 2(D)]. The fascia covering the anterior tibialis muscle was then incised, and the muscle was retracted laterally. A 3.0-mm-diameter tunnel was created in the proximal tibial metaphysis at a 30 angle relative to the long bone axis [Figure 2(E)]. A 30-mm-diameter half-circle section of PPC sheets was wrapped around the tendon as the PPC sheet treatment and fixed on the tendon with 3 - 0 Vicryl sutures [Figure 2(F)]. The length of the tunnel was measured with a depth gauge, and the

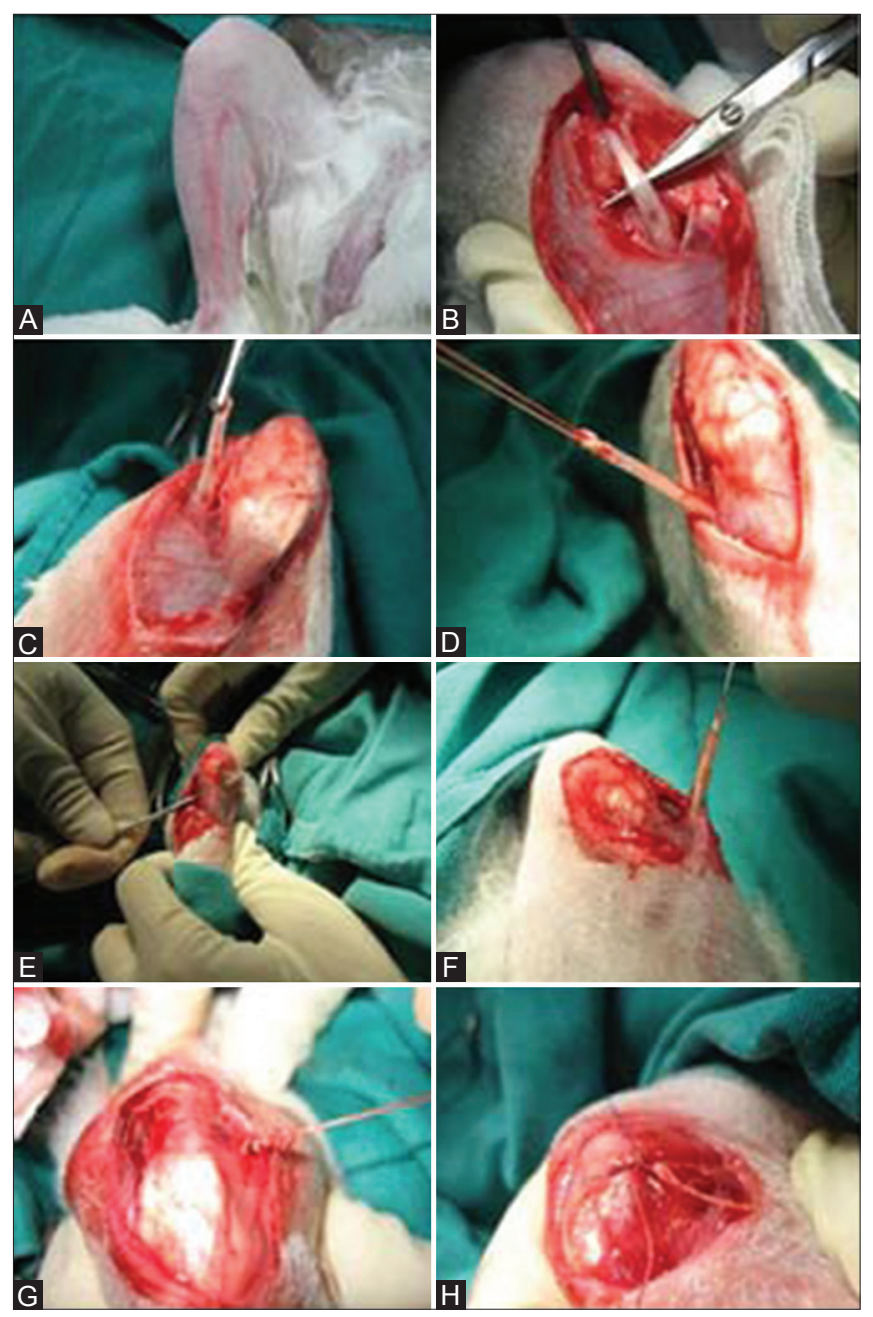

Figure 2: Surgery procedure of tendon-bone healing tunnel mode. (A) surgery position sterilization; (B) tendon isolation; (C) tendon edge cutting; (D) tendon edge suture; (E) bone tunnel creation; $(F)$ cell sheet wrapped around tendon; $(\mathrm{G})$ tendon penetrated bone tunnel; $(\mathrm{H})$ suture for fixing tendon. 
free end of the tendon was pulled manually through the drill hole [Figure 1(G)] and fixed at the medial aspect of the proximal tibia using 3 - 0 nylon suture [Figure 2(H)]. The joint capsule, fascia, and subcutaneous tissues were closed with interrupted 3 - 0 Vicryl sutures, and the skin was closed using interrupted 3 - 0 nylon sutures. The same procedure was then performed on the contralateral knee. The limbs were not immobilized postoperatively, and the rabbits were allowed to exercise as desired in individual cages. The animals were sacrificed at 4 and 8 weeks after surgery under anesthesia. The same procedure was performed in control group without PPC sheet treatment.

\section{Collagen and DNA assay}

Acid-soluble collagen content was measured with a collagen assay (Sircol; Biocolor, Belfast, Northern Ireland) according to the manufacturer's instructions using type I collagen as the standard. In brief, test samples were adjusted to $100 \mu \mathrm{L}$ in $0.5-\mathrm{M}$ acetic acid and incubated with 1-mL Sircol GAG and collagen dye reagent for $30 \mathrm{~min}$. The collagen-bound dye complex was collected by centrifugation and solubilized in $0.5-\mathrm{M}$ sodium hydroxide, and the absorbance was read at $540 \mathrm{~nm}$. The lyophilized hydrogel constructs were digested in $1 \mathrm{~mL}$ of a papainase solution $(125 \mu \mathrm{g} / \mathrm{mL}$ papain, $100 \mathrm{mM}$ PBS, $10 \mathrm{mM}$ cysteine, $10 \mathrm{mM}$ ethylenediaminetetraacetic acid, $\mathrm{pH}$ 6.3) (Worthington Biomedical, Lakewood, NJ) at $60 \mathrm{C}$ for $18 \mathrm{~h}$. Cell counts were determined by measuring the DNA content using fluorophotometry with Hoechst 33258 (Aldrich).

\section{Glycosaminoglycan assay}

Glycosaminoglycan was measured using the Blyscan-sulfated glycosaminoglycan assay (Biocolor, Belfast, Northern Ireland). Used bovine chondroitin 4-sulfate as a standard. Test samples were adjusted to $100 \mu \mathrm{l}$ mixed with $1 \mathrm{ml}$ Blyscan dye reagent for $30 \mathrm{~min}$. The glycosaminoglycan -dye complex was collected by centrifugation, bound dye was released and dissolved via addition of $1 \mathrm{ml}$ dissociation reagent and the absorbance read at $656 \mathrm{~nm}$. Glycosaminoglycan content was expressed as microgram of collagen $\mu \mathrm{g}-1$ DNA.

\section{Type II collagen detection assay}

For ELISA, test samples lysed with a lysis buffer (Sigma), centrifuged $(15,000 \times \mathrm{g}$ for $30 \mathrm{~min}$ at 4$)$, and the supernatants were subsequently analyzed. The ELISA was performed with Type II collagen detection ki (Chondrex \#6009 for type 2 collagen, Morwell Diagnostics GmbH, Zurich, Switzerland) according to the manufacturer's instructions.

\section{Alkaline phosphatase activity}

Alkaline phosphatase (ALP) activity of tendon-bone junction was assessed at 4 and 8 weeks. The tissue constructs were homogenized in $0.75 \mathrm{M}$ 2-amino-2-methylpropanol (AMP, pH 10.3; Sigma) solution, and 40-mL aliquots of the AMP supernatants were incubated with $100 \mathrm{~mL}$ of p-nitrophenyl phosphate solution at 37_C for $30 \mathrm{~min}$. The rate of p-nitrophenol production was measured at $405 \mathrm{~nm}$.

\section{Histological examination}

Ten random rabbits $(n=20$ limbs $)$ from each group during each time period were prepared for histological and immunohistochemistry evaluation of the tendon-bone tunnel, including dissection and removal of the femur and tibia with the knee joint intact. These specimens were harvested and fixed with $10 \%$ formalin for 24 hours. After decalcification and dehydration in graded alcohols, the proximal tibia was embedded in paraffin, sectioned to a thickness of $5 \mu \mathrm{m}$, and processed for in Hematoxylin-eosin (H-E), Masson's trichrome (M-T), and Safranin-O staining. The observer was blinded as to which of the two time points each sample belonged. Histological sections were observed and photographed using a light microscope.

\section{Statistical analysis}

All statistical calculations were performed with SigmaPlot statistical software version 7.0 (SPSS Inc., Chicago, IL). Differences between groups were established with a paired $t$ test. Means ( \pm standard deviations) were reported as appropriate. Values of $p=0.05$ were considered statistically significant.

\section{RESULTS}

\section{Cell distribution and sheet continuity of PPC sheet}

Complete PPC sheet was obtained after culture for 10 days in vitro [Figure 1(A)]. In confocal microscopy assay, PPC continuously spread on fibrin and monolayer formation were observed. The thickness of PPC sheet was about $20 \mu \mathrm{m}$ [Figure 1(B)].

\section{Appearance of tibia in extra-articular tendon-bone healing model}

There were 80 limbs of extra-articular tendon-bone healing model performed on 40 animals. In this study, there were no premature deaths, or joint infection. All animals were euthanized at the planned times. The average length of the tibial tunnel was about $10.0 \pm 1.0 \mathrm{~mm}$. There was no significant difference among all limbs in graft and tunnel lengths. 


\section{Histology and biochemical assay}

The biochemical assay was standardized with DNA amount. To determine whether PPC sheet results in increased matrix production, as assessed by collagen production, the PPC sheet was similar to the control group at 4- and 8-week. There were also similar result of ALP level in the PPC sheet group and control group. When analyzing the amount of collagen type II, where was more type II collagen formation in the PPC sheet group $(p=0.1)$ at 4-week. There was more type II collagen formation in the PPC sheet group compared to control group at 8-week with statistical significance $(p=0.05)$. The result of GAG showed more in PPC group at 4-week, but has no statistical significance $(p=0.16)$. The GAG decreased at 8 -week in both group, and revealed more chondrocyte formed in the PPC sheet group, but has no statistical significance $(p=0.09)$. [Figure 3]

\section{Hematoxylin-eosin staining}

Bone, fibrocartilage and collagen fiber attachment at the tendon-bone healing interface in the bone tunnel on all the studied limbs were observed [Figure 4]. Cross-sections of the bone tunnel showed that there was fibrocartilage tis-

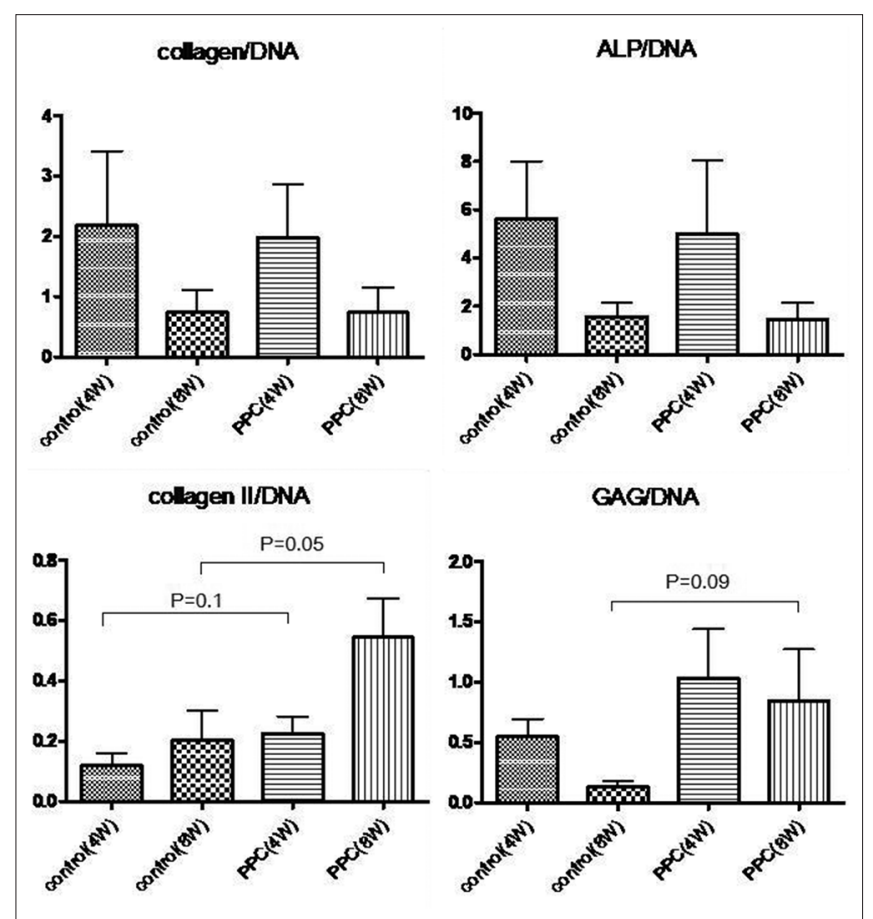

Figure 3: The total collagen amount was decreased with time goes by in control and PPC group. The ALP data was also revealed the same result. When observing the type II collagen and GAG, the PPC group has more type II collagen formation compared to the control group. The GAG in PPC group, the amount was decreased at 8 weeks than 4 weeks. It may happen because the chondrocyte might become more mature, from ovoid cell to more spindle fibrocartilage-like cell. sue formed by PPC sheet between the tendon and bone at 4 week [Figure 4(A)]. The PPC sheet treatment revealed fibrocartilage and new bone regeneration around the tendon at 8 week [Figure 4(B)]. The new bone formation was identified by the dense bone formation that was noted, which was differentiated with cancellous host bone. At 4 week, the interface between the tendon and the tibial tunnel was fused with fibrocartilage outside of the tendon and there was new bone formation between fibrocartilage and bone tunnel [Figure 4(A)]. At 8 weeks, there was

more fibrocartilage with calcified fibrocartilage formation over the tendon-bone junction. Matured fibrocartilage was fused with bone tunnel by new bone formation [Figure 4(B)]. In the control group, new bone formation lining in the bone tunnel had been interdigitated with the only fibrous interface tissue at 4 [Figure 4(C)] and 8 week [Figure 4(D)]. There were no fibrocartilage formation in control group [Figures 4(C) and (D)]. The observer was blinded to the time point and group.

\section{Masson trichrome staining}

Masson's trichrome staining more clearly showed collagen fiber and fibrocartilage formation between the tendon-bone interfaces [Figure 5]. The PPC sheet treatment revealed fibrocartilage and loose collagen fiber formation around the tendon at 4 week [Figure 5(A)]. Matured fibrocartilage and dense collagen fiber formed at the tendon-bone interface at 8 weeks [Figure 5(B)]. In control group, only collagen fiber lined the bone tunnel was observed at each time point [Figures 5(C) and (D)]. The observer was blinded to the time point and group.

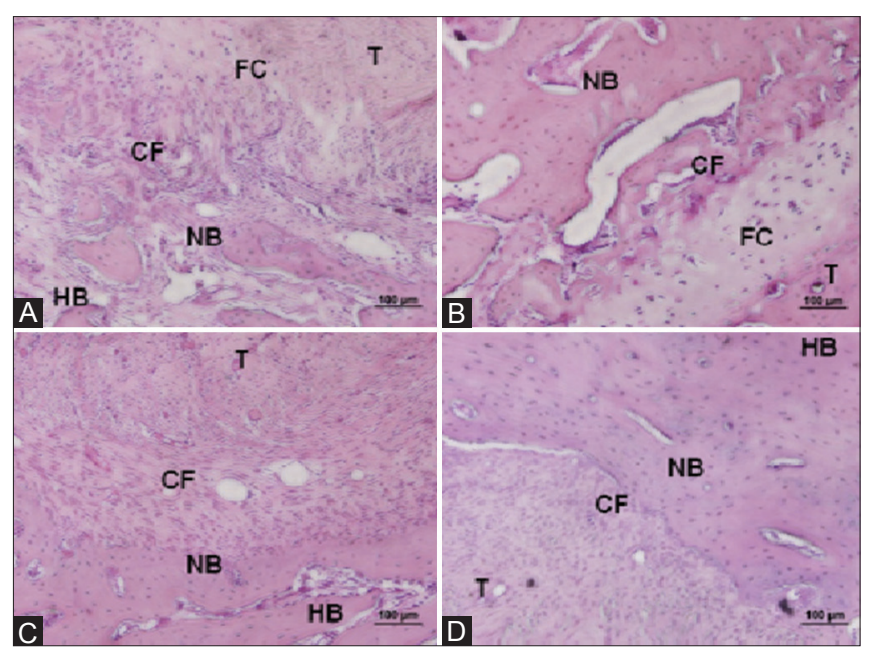

Figure 4: H-E staining show fibrocartilage tissue formation in a bone tunnel at 4 week in the PPC sheet treatment (A), and fibrocartilage and new bone regeneration around the tendon at 8 week in the PPC sheet treatment (B), at 4 week in the control group (C), at 8 week in the control group (D) postoperation. HB: Host bone; NB: New bone; CF: Collagen fiber; T: Tendon; FC: Fibrocartilage. 


\section{Safranin-O staining}

Chondrogenic differentiation assays showed that there was cartilage-like tissue appeared with the PPC sheet treatment, and positive safranin-O histochemical staining after 4 and 8 weeks [Figure 6]. At 4 weeks, the junctions between the tendon graft and the bone

tunnel were filled with lots of glycosaminoglycans in dense cells [Figure 6(A)]. Glycosaminoglycans formed in loose cells (that seemed like mature fibrocartilage tissue), was observed at 8 weeks [Figure 6(B)]. In the control group, more glycosaminoglycans deposits was found at 4 weeks [Figure 6(C)], but no glycosaminoglycans deposits was stained at 8 weeks [Figure 6(D)]. The observer was blinded to the time point and group.

\section{DISCUSSION}

Tendon-bone healing in a bone tunnel occurs initially by granulation tissue in the interface between the bone and the tendon along the bone tunnel. With time goes by, the progressive increase in strength was correlated with the degree of bone ingrowths, mineralization, and maturation of the healing tissue. ${ }^{[1]}$ Previous studies describe the tendon-bone healing with the order of a period of highly cellular and collagen fiber scaring tissue, and then reorganized to a dense connective tissue matrix. Sharpey's fibers were appeared later ${ }^{[22]}$ Progressive re-establishment of collagen fiber continuity between the tendon and the bone facilitates re-establishment of a tendo-osseous junction. Goradia at el had proved that perpendicular and circumferential collagen fibers were connected the tendon graft to the bone tunnels

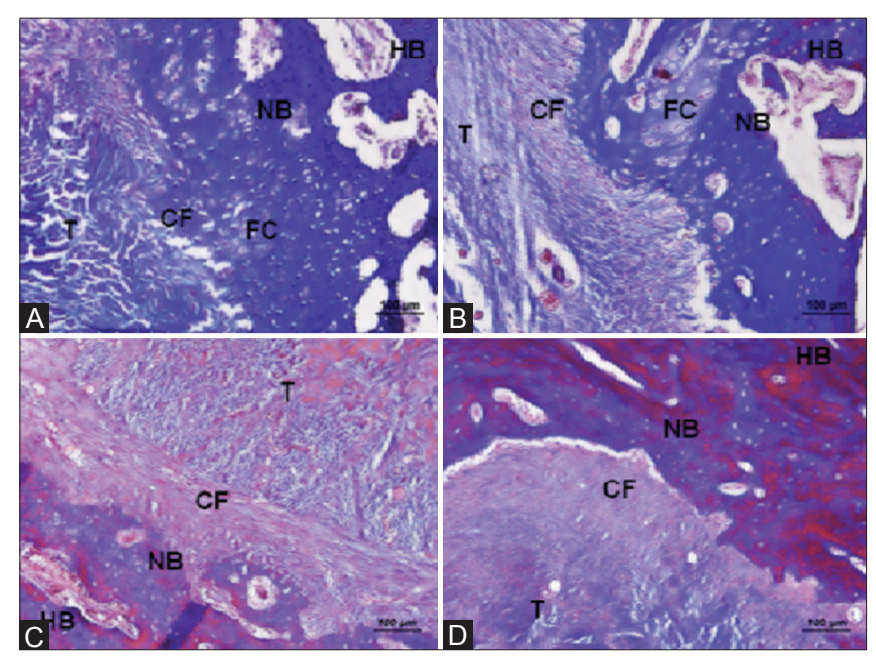

Figure 5: M-T staining show fibrocartilage and loosen collagen fiber formation around the tendon at 4 week (A), and matured fibrocartilage and dense collagen fiber formatted at 8 week (B), at 4 week in the control group (C), at 8 week in the control group (D) postoperation. HB: Host bone; NB: New bone; CF: Collagen fiber; T: Tendon; FC: Fibrocartilage. in sheep animal model. ${ }^{[23]}$ The development of Sharpey-like collagen fibers connecting the tendon graft and the bone tunnel has been described and viewed as the earliest sign of osteointegration. ${ }^{[11,22]}$

Focus on the histology of normal ACL structure, the tendon bone attachment was formed by the intermediate zone of fibrocartilage. ${ }^{[24,25]}$ According to this, the ideal attachment of tendon graft and bone tunnel would be the fibrocartilage. In ACL reconstruction, when we examine the failure case that reconstructed with hamstring tendon, the insertions of hamstring autografts to the bone tunnel was composed with fibrous tendon insertion by immunostaining. ${ }^{[3]}$ For that reason, we do not know why that fibrous tendon insertion formation in some cases and it also influence the overall result of ACL reconstruction. For better tendon bone healing, previous study had showed that when ACL reconstruction with the periosteum-enveloped tendon, the periosteum could form the fibrocartilage at the interface of tendon graft and bone tunnel with its progenitor cell in rabbit model..$^{[16,17]}$

The periosteum consists of multipotent mesodermal cells that are capable of differentiating into various types of connective tissue and bone. ${ }^{[11,12]}$ Experimental studies have revealed that progenitor cells in free periosteal grafts will be inducted by surrounding tissue. ${ }^{[14,15,17]}$ Histological examination of the periosteum revealed the presence of an outer "fibrous layer" and an inner "cambium layer". The fibrous layer contains fibroblasts and the cambium layer contains progenitor cells..$^{[12]}$ By attaching the periosteum onto the tendon-bone interface, the progenitor cells in the cambium layer can differentiate into osteoblasts and chondroblasts and subsequently into fibrocar-

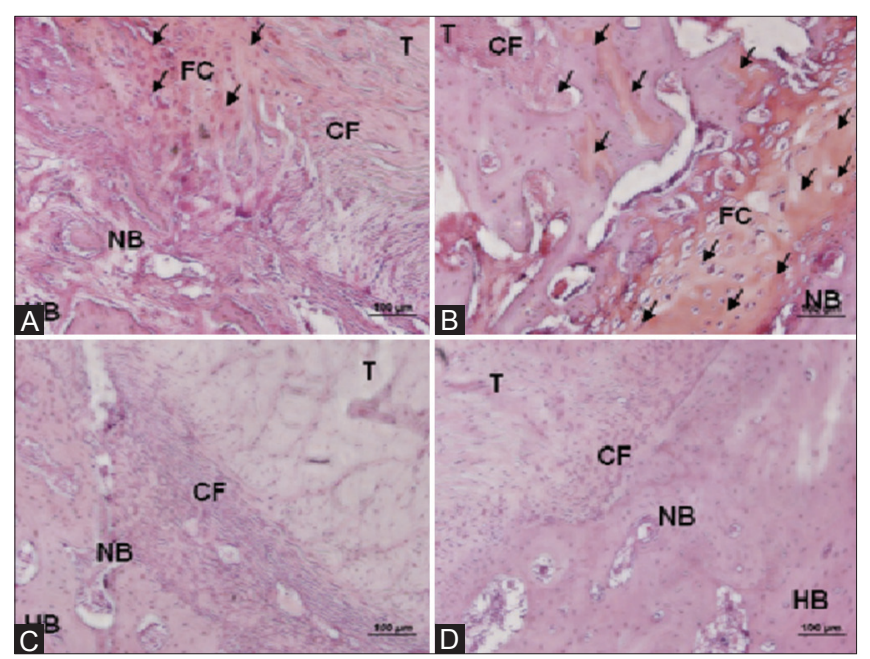

Figure 6: HandSafranin O staining show glycosaminoglycans in dense cells between tendon and bone at 4 week (A), and glycosaminoglycans formation in loose cells at 8 week (B), at 4 week in the control group (C), at 8 week in the control group (D) post-operation. HB: Host bone; NB: New bone; CF: Collagen fiber; T: Tendon; FC: Fibrocartilage. Black arrow indicates glycosaminoglycans. 
tilage and calcified fibrocartilage. The progenitor cells in the cambium layer of the periosteum can facilitate bone ingrowth into the collagenous tissue formed by the fibroblasts in the fibrous layer of the periosteum, and can induce ossification and bone formation. ${ }^{[11]}$ In the rabbit model, the periosteum was applied to tendon grafts by enveloping the tendon in a bone tunnel. ${ }^{[11,16]}$ Histological examinations revealed that bone ingrowth into the cambium layer appeared at 4 weeks, and that interdigitation occurred between the periosteum tissue and the tendon. The fibrous layer from the wrapped periosteum progressively became a collagen fiber-bone intermixture and anchorage, and incorporation and organization on the interface developed with time. Biomechanical testing demonstrated a progressive increase in tendon pull-out strength followed by bone ingrowth, mineralization, and incorporation of the healing tissue, and revealed a statistically significant difference at 8 and 12 weeks. It appears that the periosteum offers a powerful inductive ability between the tendon and the bone tunnel to incorporate healing.

Biomimetic materials produced by tissue engineering have been developed for filling the interface of the repair site. Combining the stimulation of the signal growth factor and material could induce cellular chemotaxis, proliferation, differentiation, and notably new tissue formation at a required site. In 1993, Okano at el developed a novel system for cultured cells using plasma-treated polystyrene dishes grafted with poly (N-isopropylacrylamide). ${ }^{[19]}$ This technique which was using temperature-responsive culture dishes allows the noninvasive harvest of cultured cells as intact sheets with their formatted extracellular matrix. The cell sheets can be directly transplanted to host tissues without scaffolds, carrier materials, or tissue constructs via the layering of individual cell sheets. ${ }^{[20]}$ The technique can apply for some tissue reconstructions, including heart, ${ }^{[21,26,27]}$ skeletal muscle, ${ }^{[28]}$ and cornea. ${ }^{[29]}$ Cell sheet also could be formatted by a scaffold-free method using biodegradable polymerized fibrin-coated dishes. Polymerized fibrin-coated dishes were prepared with human fibrinogen monomers mixed with thrombin. The cell sheets were easily dissociated intact from the polymerized fibrin layer. This simple method of cell sheet engineering may also be applicable for various other cell types. ${ }^{[21]}$

We used this concept of periosteal progenitor cell and an intact cell sheet without scaffolds or carrier materials to develop a cell sheet composed periosteal progenitor cell. We use the cell sheets to enhance tendon bone healing. The result demonstrated improvement of healing by applying the PPC sheet at the tendon-bone interface. Histological analysis in this study of the PPC sheet revealed an increased amount of glycosaminoglycans and perpendicular collagen fibers at the insertion. We also found that promoted fibrocartilaginous attachment and bone neoformation in the PPC sheet in extra-ar- ticular tendon-bone healing model in rabbits. The biochemical assay revealed no difference between collagen and ALP, but all decreased with time goes by. When examine the amount of type II collagen, we could find that significant increased type II collagen in PPC group. The result of GAG also revealed that more GAG in PPC group. Combined the data of biochemical assay, we think that the tendon-bone interface became stable from 4-week to 8-week, which was revealed by total collagen and ALP. When focusing on type II collagen, we could see that the amount of type II collagen was much more in PPC group, especially at 8-week. We thought that more chondrocyte was formed in PPC group, which could become mature fibrocartilage at tendon-bone interface. The amount GAG in PPC group at 8-week was slightly decreased, and we thought that may because of maturation of fibrocartilage. The histological examine could reveal the morphology of tendon-bone interface. Therefore, the presence of fibrocartilage tissue in this study could have strengthened attachment at the tendon-bone interface. Histological examination revealed progressive bone ingrowth, mineralization, and incorporation of healing tissue over time. The PPC sheet treatment enhanced tendon-bone healing with fibrocartilage formation as early as 4 week. This means that, with PPC sheet-treated tendon-bone interface, there were progressive chondrocyte formations which lead to fibrocartilage. The PPC sheet could enhance the formation of fibrocartilage, which could limit tunnel enlargement and improve the healing response of the graft in the tunnel.

In this study, it appears that the PPC sheet possesses a powerful inductive ability between tendon and bone to incorporate healing. This technique may provide a novel platform for tissue-engineered stem cell therapies in enhancing tendon bone healing. The PPC sheet could be applied to any situation in which a tendon graft is placed into bone tunnel, such as in ulnar collateral ligament reconstruction in the elbow, coracoclavicular ligament reconstruction, where there were no periosteum to harvest; and ACL and PCL reconstruction in the knee. Furthermore, the PPC sheet produces an impressive enhancement of tendon-bone healing, a minimally invasive clinical consideration via arthroscopy of tissue regeneration.

The limitations of this study include that this is an extra-articular model to evaluate the tendon bone healing after tendon reconstruction, so the environment of tendon bone healing was different. There were no joint fluid and joint movement that could inference the tendon bone healing in our study. However, histological examine of the tendon-bone interface could demonstrate the good tendon-bone healing enhanced by PPC sheet with fibrocartilage formation.

\section{Conclusions}

Periosteal progenitor cell monolayer maintains the differentiated capacity and osteochondral potential which could promote fibrocartilage formation in tendon-bone junction. 
Bioengineered PPC sheets can offer a new feasible therapeutic strategy of a novel approach to enhance tendon-bone junction healing.

\section{Acknowledgments}

The authors would like to thank the National Science Council (grant- NSC96 - 2314-B-182A-054-MY3) for financial support of this research. The authors also acknowledge the staff of the Medical Research Center, the Laboratory Animal Center, and the Confocal Laser Scanning Microscopic Room at Chang Gung Memorial Hospital at Keelung for their technical assistance.

\section{REFERENCES}

1. Rodeo SA, Arnoczky SP, Torzilli PA, Hidaka C, Warren RF. Tendon-healing in a bone tunnel. A biomechanical and histological study in the dog. J Bone Joint Surg Am 1993;75:1795-803.

2. Oguma H, Murakami G, Takahashi-Iwanaga H, Aoki M, Ishii S. Early anchoring collagen fibers at the bone-tendon interface are conducted by woven bone formation: Light microscope and scanning electron microscope observation using a canine model. J Orthop Res 2001;19:873-80.

3. Petersen W, Laprell H. Insertion of autologous tendon grafts to the bone: A histological and immunohistochemical study of hamstring and patellar tendon grafts. Knee Surg Sports Traumatol Arthrosc 2000;8:26-31.

4. Chen CH. Graft healing in anterior cruciate ligament reconstruction. Sports Med Arthrosc Rehabil Ther Technol 2009;1:21.

5. Hashimoto Y, Yoshida G, Toyoda H, Takaoka K. Generation of tendon-to-bone interface "enthesis" with use of recombinant BMP-2 in a rabbit model. J Orthop Res 2007;25:1415-24.

6. Yamazaki S, Yasuda K, Tomita F, Tohyama H, Minami A. The effect of transforming growth factor-beta1 on intraosseous healing of flexor tendon autograft replacement of anterior cruciate ligament in dogs. Arthroscopy 2005;21:1034-41.

7. Martinek V, Latterman C, Usas A, Abramowitch S, Woo SL, Fu FH, et al. Enhancement of tendon-bone integration of anterior cruciate ligament grafts with bone morphogenetic protein-2 gene transfer: A histological and biomechanical study. J Bone Joint Surg Am 2002;84-A: 1123-31.

8. Harris MT, Butler DL, Boivin GP, Florer JB, Schantz EJ, Wenstrup RJ. Mesenchymal stem cells used for rabbit tendon repair can form ectopic bone and express alkaline phosphatase activity in constructs. J Orthop Res 2004;22:998-1003.

9. Ouyang HW, Goh JC, Lee EH. Use of bone marrow stromal cells for tendon graft-to-bone healing: Histological and immunohistochemical studies in a rabbit model. Am J Sports Med 2004;32:321-7.

10. Ju YJ, Muneta T, Yoshimura H, Koga H, Sekiya I. Synovial mesenchymal stem cells accelerate early remodeling of tendon-bone healing. Cell Tissue Res 2008;332:469-78.

11. Ritsila VA, Santavirta S, Alhopuro S, Poussa M, Jaroma H, Rubak JM, et al. Periosteal and perichondral grafting in reconstructive surgery. Clin Orthop Relat Res 1994;302:259-65.
12. Augustin G, Antabak A, Davila S. The periosteum. Part 1: Anatomy, histology and molecular biology. Injury 2007;38:1115-30.

13. Ritsila V, Alhopuro S, Rintala A. Bone formation with free periosteum. An experimental study. Scand J Plast Reconstr Surg $1972 ; 6: 51-6$

14. Rubak JM. Osteochondrogenesis of free periosteal grafts in the rabbit iliac crest. Acta Orthop Scand 1983;54:826-31.

15. Uddstromer L, Ritsila V. Osteogenic capacity of periosteal grafts. A qualitative and quantitative study of membranous and tubular bone periosteum in young rabbits. Scand J Plast Reconstr Surg $1978 ; 12: 207-14$

16. Chen CH, Chen WJ, Shih CH, Yang CY, Liu SJ, Lin PY. Enveloping the tendon graft with periosteum to enhance tendon-bone healing in a bone tunnel: A biomechanical and histologic study in rabbits. Arthroscopy 2003;19:290-6.

17. Youn I, Jones DG, Andrews PJ, Cook MP, Suh JK. Periosteal augmentation of a tendon graft improves tendon healing in the bone tunnel. Clin Orthop Relat Res 2004:223-31.

18. Chen $\mathrm{CH}$, Chen WJ, Shih $\mathrm{CH}$, Chou SW. Arthroscopic anterior cruciate ligament reconstruction with periosteum-enveloping hamstring tendon graft. Knee Surg Sports Traumatol Arthrosc 2004;12:398-405.

19. Okano T, Yamada N, Sakai H, Sakurai Y. A novel recovery system for cultured cells using plasma-treated polystyrene dishes grafted with poly (N-isopropylacrylamide). J Biomed Mater Res 1993;27:1243-51.

20. Yang J, Yamato M, Nishida K, Ohki T, Kanzaki M, Sekine H, et al. Cell delivery in regenerative medicine: The cell sheet engineering approach. J Control Release 2006;116:193-203.

21. Itabashi Y, Miyoshi S, Kawaguchi H, Yuasa S, Tanimoto K, Furuta A, et al. A new method for manufacturing cardiac cell sheets using fibrin-coated dishes and its electrophysiological studies by optical mapping. Artif Organs 2005;29:95-103.

22. Liu SH, Panossian V, Al-Shaikh R, Tomin E, Shepherd E, Finerman GA, et al. Morphology and matrix composition during early tendon to bone healing. Clin Orthop Relat Res 1997;339:253-60.

23. Goradia VK, Rochat MC, Grana WA, Rohrer MD, Prasad HS. Tendon-to-bone healing of a semitendinosus tendon autograft used for ACL reconstruction in a sheep model. Am J Knee Surg 2000;13:143-51.

24. Cooper RR, Misol S. Tendon and ligament insertion. A light and electron microscopic study. J Bone Joint Surg Am 1970;52:1-20.

25. Benjamin M, Evans EJ. Fibrocartilage. J Anat 1990;171:1-15.

26. Itabashi Y, Miyoshi S, Yuasa S, Fujita J, Shimizu T, Okano T, et al. Analysis of the electrophysiological properties and arrhythmias in directly contacted skeletal and cardiac muscle cell sheets. Cardiovasc Res 2005;67:561-70.

27. Masuda S, Shimizu T, Yamato M, Okano T. Cell sheet engineering for heart tissue repair. Adv Drug Deliv Rev 2008;60:277-85.

28. Koning M, Harmsen MC, van Luyn MJ, Werker PM. Current opportunities and challenges in skeletal muscle tissue engineering. J Tissue Eng Regen Med 2009;3:407-15.

29. Nakamura T, Kinoshita S. Current regenerative therapy for the cornea. Nippon Rinsho 2008;66:955-60. 